\title{
The Aramis Data Challenge: Prognostics and Health Management in Evolving Environments
}

\author{
Francesco Cannarile
}

Aramis Srl, Italy.

Energy Department, Politecnico di Milano, Italy.E-mail: francesco.cannarile@aramis3d.com

Michele Compare

Aramis Srl, Italy.

Energy Department, Politecnico di Milano, Italy.E-mail: michele.compare@aramis3d.com

Piero Baraldi

Energy Department, Politecnico di Milano, Italy.E-mail: piero.baraldi@polimi.it

Zhe Yang

Aramis Srl, Italy.

School of Mechanical Engineering, Dongguan University of Technology, Dongguan, 523808, China.

E-mail: zhe.yang@polimi.it

\section{Enrico Zio}

Aramis Srl, Italy.

Energy Department, Politecnico di Milano, Italy.

MINES ParisTech, PSL Research University, France.

Department of Nuclear Engineering, Kyung Hee University, Republic of Korea. E-mail: enrico.zio@polimi.it

The objective of the Aramis Data Challenge is the creation of a public benchmark dataset for the problem of fault detection in evolving environments. A multi-component system in which the degradation of one component accelerates the degradation processes of the other components, thus modifying their lifetime distributions and the statistical properties of the monitored signals over time is considered. Here, we provide details with respect to the Challenge definition, the data collection and the evaluation metric.

\section{Symbols}

\begin{tabular}{|c|c|}
\hline$J$ & $\begin{array}{l}\text { Number of components in an } \\
\text { industrial system }\end{array}$ \\
\hline$T$ & $\begin{array}{l}\text { Mission time of the industrial } \\
\text { system }\end{array}$ \\
\hline$M$ & Number of industrial systems \\
\hline$j$ & Index of the component \\
\hline$m$ & Index of the industrial system \\
\hline$D_{t}^{j, m}$ & $\begin{array}{l}\text { The degradation level of the } j^{t h} \\
\text { component in the } m^{t h} \text { system at } \\
\text { time } t\end{array}$ \\
\hline$d_{f}$ & $\begin{array}{l}\text { Threshold defining the abnormal } \\
\text { condition of a component }\end{array}$ \\
\hline$T_{f}$ & $\begin{array}{l}\text { The time at which the system } \\
\text { failure occurs }\end{array}$ \\
\hline$K$ & $\begin{array}{l}\text { Number of sensors installed on a } \\
\text { component }\end{array}$ \\
\hline
\end{tabular}

$\begin{array}{cl}s & \text { Signal measurement } \\ E_{t}^{m} & \text { Operating condition of the } m^{t h} \\ & \text { system at time } t \\ f_{s} & \text { Sampling frequency } \\ \boldsymbol{x}_{t} & \quad \text { The vector of } K \text { measurements } \\ & \text { taken from a component at time } t \\ y_{t} & \text { The binary label indicating } \\ & \text { normal/abnormal conditions of a } \\ & \text { component } \\ \tau & \text { The time at which a component } \\ & \text { enters into an abnormal condition } \\ D_{\text {train }} & \text { Training set } \\ T_{\text {life }}^{m} & \text { Lifetime of the } m^{\text {th }} \text { system } \\ D_{\text {test }} & \text { Test set } \\ M_{\text {test }} & \text { Number of systems in the test set } \\ \hat{\tau} & \text { Estimation of the onset time of the } \\ & \text { abnormal condition }\end{array}$


$\Delta \quad$ Error with respect to the estimation of onset of abnormal condition

\section{$A$}

The proposed metric for performance evaluation

$a_{1}, a_{2}, b_{1}, b_{2} \quad$ Parameters used for metric calculation

\section{Introduction}

Prognostic and Health Management (PHM) is an innovative, interdisciplinary research topic with the objective of detecting and diagnosing the abnormal behaviors of industrial systems and predict their future behavior. The capability of performing these PHM tasks with sufficient accuracy provides the opportunity of avoiding system unscheduled shutdowns, setting efficient maintenance strategies to fully exploit the useful life of the industrial system, with significant potential advantages on system safety, availability and cost savings (Elghazel et al (2015); Zio (2013)).

A recurrent difficulty for the effective application of PHM tools in practice is related to the "evolving environment" in which industrial components typically operate, due to deterioration of components and sensors, maintenance activities, upgrading plans involving new components and system architectures, and the change in the operational and environmental conditions. Since industrial systems are getting more and more complex, the issue of evolving environment becomes even more complicated in case of multi-component systems, due to the (stochastic) dependence of the components degradation processes (Bian and Gebraeel (2014); Dekker et al. (1997)): the degradation of one component can accelerate the degradation processes of the other components, thus modifying their lifetime distributions and the statistical properties of the monitored signals over time. To name a few examples, the degradation of hydrodynamic bearings in wind turbines may lead to increasing the looseness of primary transmission shafts, which in turn increases the vibration levels in the gearbox and accelerate the degradation of the gears (Bian and Gebraeel (2014)). In power grids, the aging of generators or transformers in a subnetwork may lead to the overloading of the other units in the network and accelerating their degradation (Bian and Gebraeel (2014)). And in the industrial cold box used in petrochemical plants, the extent of fouling of one tube of the cold box can accelerate the fouling rate of other tubes due to overloading (Rasmekomen and Parlikad (2016)).

As an important task of PHM, fault detection aims to promptly identify the onset of abnormal condition of the industrial system. In recent years, various datasets become publicly available for the verification of fault detection methods, in order to enable easier exchange, participation and collaboration with the scientific community. These datasets can be classified into those obtained by simulating the operation of industrial equipment using physics-based models and those containing real signal measurements. The former class includes datasets simulating the operation of a ship propulsion system (Izadi-Zamanabadi and Blanke (1999)), a valve actuator system (Syfert et al. (2003)), an electro-mechanical position servo system (Blanke et al. (1995)) and a wind turbine system (Toubakh and Sayed-Mouchaweh (2015)). Since the fault scenarios are artificially created, the time of the onset of the abnormal conditions is known and, therefore, the performance of the fault detection methods can be precisely estimated. However, differently from real-world situations, they typically contain a small number of signals and they are not able to realistically reproduce the evolving environments. The latter class includes datasets containing real signal measurements collected from bearings (Qiu et al. (2006)), gearboxes (https://www.phmsociety.org/references/datasets ), anemometers (https://www.phmsociety.org/competition/phm/1 1), power plants (https://www.phmsociety.org/events/conference/ $\mathrm{phm} / 15 /$ data-challenge) and vehicles (Jia et al. (2018);

https://www.phmsociety.org/events/conference/p $\mathrm{hm} / 17 /$ data-challenge). These datasets contain various types of faults of a specific component (system) in different working conditions. Their use for the verification of fault detection methods is, however, complicated by the fact that the true time of the onset of the abnormal conditions is typically not known. Also, several of these datasets focus on a single component and do not consider one of the most common causes of evolving environments, which is the effects on the monitored component of the degradation and failure of other components.

Given the limitations of the available datasets for the validation of fault detection methods, and in an effort to convey research towards the solution of this key practical hurdle for PHM application in evolving environments, we present a novel benchmark in which the presence of evolving environment is simulated considering the effects of the degradation and failures of other components on the monitored one, whose normal/abnormal state is known. The availability of the ground-truth model allows fairly assessing the proposed PHM solutions. 

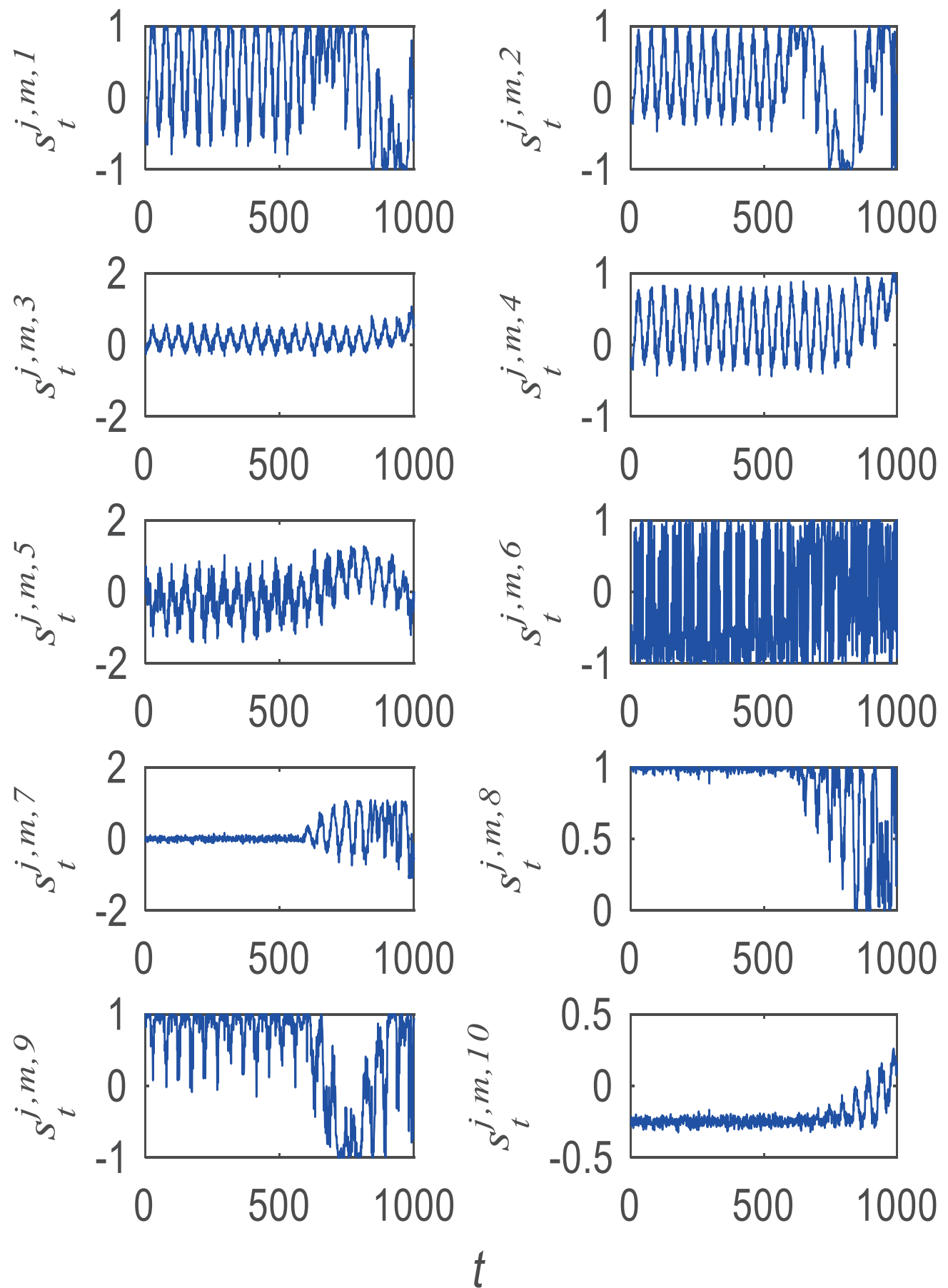

Fig. 1. Evolution of the 10 monitoring signals during a component life. 


\section{Data and Ground Truth}

The industrial system of interest is made up of $J=4$ interconnected identical components, with mission times $T \in \mathbb{R}_{+}$, in arbitrary time units (atu). A number $M$ of four component systems are installed in $M$ different plants. The degradation of the $j^{\text {th }}$ component, $j=1, \ldots, 4$, in the $m^{t h}, m=1, \ldots, M$, system is modeled as a time-continuous stochastic process $D^{j, m}=$ $\left\{D_{t}^{j, m}\right\}_{t \geq 0}$ defined on a probability space $(\Omega, \mathcal{F}, \mathbb{P})$. A component enters the abnormal condition when $D_{t}^{j, m}$ exceeds a threshold $d_{f}$. This abnormal condition state does not correspond to the component failure, but makes the operating conditions of the system harsher, which in turn accelerates the degradation process of the other components of the same system. The system failure occurs at time $T_{f}$, when all four components start operating in abnormal conditions. The degradation paths of the four components of all $M$ systems have been simulated up to either the mission time $T$ or the times of system failures, whichever comes first.

The level of degradation of the components can be estimated through $K=10$ sensors installed on each component. The sensor signals, $s_{t}^{j, m, 1}, \ldots, s_{t}^{j, m, K}$, are influenced by both the degradation levels $D_{t}^{j, m}, j=1, \ldots, 4$, and the operating condition $E_{t}^{m}$. The measurements are taken at a fixed frequency $f_{s}=1 \mathrm{atu}^{-1}$.

An example of the evolutions of the $K$ signals of a component in a given system is shown in Fig. 1.

\section{Problem Statement}

Let $\boldsymbol{x}_{t}^{j, m}=\left[s_{t}^{j, m, 1}, \ldots, s_{t}^{j, m, K}\right] \in \mathbb{R}^{K}$ be the vector of the $K$ measurements taken from the $j^{\text {th }}$ component of the $m^{\text {th }}$ system at time $t$ and $y_{t}^{j, m} \in\{0,1\}$ the label of the component with 0 indicating normal conditions and 1 indicating abnormal conditions at time $t$. Let $\tau^{j, m} \in \mathbb{R}^{+}$be the time of the first entry into an abnormal state, i.e., $y_{t}^{j, m}=0$ when $t<\tau^{j, m}$ and $y_{t}^{j, m}=1$ when $t \geq \tau^{j, m}$. Notice that it can occur that $\tau^{j, m}>T$.

Given a training set of degradation trajectories:

$$
\begin{gathered}
D_{\text {train }}=\left\{\left[\boldsymbol{x}_{t}^{1, m}, \ldots, \boldsymbol{x}_{t}^{J, m}\right],\left[y_{t}^{1, m}, \ldots, y_{t}^{J, m}\right],\right. \\
\left.t=0, \ldots, T_{\text {life }}^{m}\right\}, m=1, \ldots, M
\end{gathered}
$$

where:

$$
T_{\text {life }}^{m}=\min \left(T, T_{f}^{m}\right), m=1, \ldots, M
$$

The objective of this challenge is to develop a model to identify the onset of the abnormal condition of all $J$ components in a test system $D_{\text {test }}=\left\{\left[\boldsymbol{x}_{t}^{1, \text { test }}, \ldots, \boldsymbol{x}_{t}^{J, \text { test }}\right], t=0, \ldots, T_{\text {life }}^{\text {test }}\right\}$.

To this aim, the degradation trajectories of 250 four-component systems have been simulated. The obtained dataset has been randomly partitioned into a labelled training set containing the degradation trajectories of $M=$ 200 systems for the development of a model for the identification of the onset of abnormal conditions and an unlabelled test set of $M_{\text {test }}=$ 50 systems to validate the performance of the developed model.

\section{Performance Metric}

The test set containing the degradation trajectories of $M_{\text {test }}=50$ four-component systems is considered for the evaluation of the performance. The following quantities are introduced:

- $\quad T_{\text {life }}^{m}$ : lifetime of the $m^{\text {th }}$ system, defined by Eq. (2);

- $\tau^{j, m}$ : ground truth time of the first entry of the $j^{\text {th }}$ component of the $m^{\text {th }}$ system into an abnormal state. In the case in which the component does not enter into an abnormal state within the time horizon $T_{\text {life }}^{m}, \tau^{j, m}$ is set to NaN (Not A Number).

An estimate $\hat{\tau}^{j, m}$ of $\tau^{j, m}$ is required for any component, $j=1, \ldots, J$, of any system, $m=$ $1, \ldots, M_{\text {test }}$. If no entry into an abnormal state is detected, $\hat{\tau}^{j, m}$ should be set to NaN.

The error that one makes in estimating the time $\tau^{j, m}$ with $\hat{\tau}^{j, m}$ is defined by:

$$
\begin{aligned}
& \Delta^{j, m}= \\
& \left\{\begin{array}{cl}
\tau^{j, m}-\hat{\tau}^{j, m} & \tau^{j, m} \neq \mathrm{NaN}, \hat{\tau}^{j, m} \neq \mathrm{NaN} \\
0 & \tau^{j, m}=\mathrm{NaN}, \hat{\tau}^{j, m}=\mathrm{NaN} \\
k_{\text {false }} & \tau^{j, m}=\mathrm{NaN}, \hat{\tau}^{j, m} \neq \mathrm{NaN} \\
-k_{\text {missed }} & \tau^{j, m} \neq \mathrm{NaN}, \hat{\tau}^{j, m}=\mathrm{NaN}
\end{array}\right. \\
& j=1, \ldots, J ; m=1, \ldots, M
\end{aligned}
$$

Notice that an error equal to $k_{\text {false }}>T$ is assigned in case of false alarms (i.e., $\tau^{j, m}=$ $\mathrm{NaN}, \hat{\tau}^{j, m} \neq \mathrm{NaN}$ ) and an error of $k_{\text {missed }}>T$ is assigned in case of missed alarms (i.e., $\tau^{l} \neq$ $\mathrm{NaN}, \hat{\tau}^{l}=\mathrm{NaN}$ ).

The following metric is used to quantify the average error of the solution provided by the challenge participant on all test components: 


$$
A=\frac{1}{4 M_{\text {test }}} \sum_{m=1}^{M_{\text {test }}} \sum_{j=1}^{4} \varphi\left(\Delta^{j, m}\right) \in[0,1]
$$

with:

$$
\begin{gathered}
\varphi\left(\Delta^{j, m}\right)= \\
\left\{\begin{array}{cc}
1 & \Delta^{j, m}<-T \\
\left(1-e^{\Delta^{j, m} / a_{1}}\right) b_{1} & -T \leq \Delta^{j, m}<0 \\
\left(1-e^{-\Delta^{j, m} / a_{2}}\right) b_{2} & 0 \leq \Delta^{j, m} \leq T \\
1 & \Delta^{j, m}>T \\
j=1, \ldots, J ; m=1, \ldots, M
\end{array}\right.
\end{gathered}
$$

and

$$
\begin{aligned}
& b_{1}=1 /\left(1-e^{-T / a_{1}}\right) \\
& b_{2}=1 /\left(1-e^{-T / a_{2}}\right)
\end{aligned}
$$

Parameters $b_{1}$ and $b_{2}$ are set to obtain $\varphi(T)=$ 1 and $\varphi(-T)=1$, respectively. Parameters $a_{1}$ and $a_{2}$ are set equal to 10 and 13 , respectively, in such a way that delayed estimates are more penalized than anticipated estimates (Saxena et al. (2008)). As shown in Fig. 2, the value of metric $A$ increases with $\left|\Delta^{\jmath, m}\right|$, whereas the evolution of $A$ is faster when $\Delta^{j, m}<0$ to more penalize delayed than anticipated estimates.

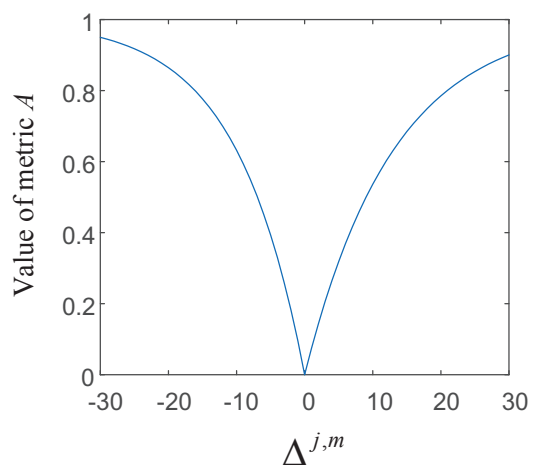

Fig. 2. Value of the proposed metric $A$ with respect to the error $\Delta^{j, m}$.

The proposed metric $A$ is a variant of the timeliness metric (Saxena et al. (2008)) that has been used in the PHM08 data challenge (Saxena and Goebel (2008); Ramasso (2014)) to sort participants' algorithm. Finally, we observe that this metric is desired to be as small as possible (ideally close to 0 ).

\section{Outlook}

The Aramis Data Challenge proposed within the ESREL2020-PSAM15 conference serves to inspire the interests of numerous researchers to come forward with their valuable contributions to the field of fault detection in evolving environments and to show to industry the potentiality of the available methods for fault detection in practical applications. Special thanks go to all Challenge participants for their hard work.

\section{References}

Bian L, Gebraeel N. Stochastic modeling and real-time prognostics for multi-component systems with degradation rate interactions. IIE Trans (Institute Ind Eng 2014;46:470-82. https://doi.org/10.1080/0740817X.2013.812269.

Blanke M, Bøgh SA, Jørgensen RB, Patton RJ. Fault detection for a diesel engine actuator - A benchmark for FDI. Control Eng Pract 1995;3:1731-40. https://doi.org/10.1016/09670661(95)96891-R.

Dekker R, Wildeman RE, van der Duyn Schouten FA. A review of multi-component maintenance models with economic dependence. Math Methods Oper Res 1997;45:411-35. https://doi.org/10.1007/BF01194788.

Elghazel W, Bahi J, Guyeux C, Hakem M, Medjaher K, Zerhouni N. Dependability of wireless sensor networks for industrial prognostics and health management. Comput Ind 2015;68:1-15.

Izadi-Zamanabadi R, Blanke M. A ship propulsion system as a benchmark for fault-tolerant control. Control Eng Pract 1999;7:227-39. https://doi.org/10.1016/S0967-0661(98)00149-X.

Jia X, Huang B, Feng J, Cai H, Lee J. Review of PHM data competitions from 2008 to 2017: Methodologies and analytics. Proc. Annu. Conf. Progn. Heal. Manag. Soc. PHM, 2018.

Qiu H, Lee J, Lin J, Yu G. Wavelet filter-based weak signature detection method and its application on rolling element bearing prognostics. J Sound Vib 2006;289:1066-90. https://doi.org/10.1016/j.jsv.2005.03.007.

Rasmekomen N, Parlikad AK. Condition-based maintenance of multi-component systems with degradation state-rate interactions. Reliab Eng Syst Saf 2016;148:1-10.

Ramasso E. Investigating computational geometry for failure prognostics. 2014.

Syfert M, Patton R, Bartyś M, Quevedo J. Development and application of methods for actuator diagnosis in industrial control systems (DAMADICS): A benchmark study. IFAC Proc. Vol., vol. 36, 2003, p. $\quad 843-54$. https://doi.org/10.1016/S14746670(17)36598-9.

Saxena A, Celaya J, Balaban E, Goebel K, Saha B, Saha $\mathrm{S}$, et al. Metrics for Evaluating Performance of Prognostic Techniques. 2008 Int. Conf. Progn. Heal. Manag., 345 E 47TH ST, NEW YORK, NY 10017 USA: IEEE; 2008, p. 174+.

Saxena A, Goebel K. Phm08 challenge data set. NASA Ames Progn Data Repos 2008. 
Proceedings of the 30th European Safety and Reliability Conference and the 15th Probabilistic Safety Assessment and Management Conference

Toubakh H, Sayed-Mouchaweh M. Hybrid dynamic data-driven approach for drift-like fault detection in wind turbines. Evol Syst 2015;6:115-29. https://doi.org/10.1007/s12530-014-9119-8.

Zio E. Prognostics and health management of industrial equipment. Diagnostics Progn. Eng. Syst. methods Tech., IGI Global; 2013, p. 333-56. 